\author{
Military Technical College \\ Kobry Elkobbah, \\ Cairo, Egypt \\ May $16-18,2006$
}

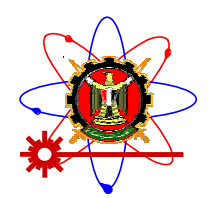

$3^{\text {nd }}$ International Conference on

Engineering Mathematics and

Physics (ICMEP-3)

\title{
HIGHER ORDERS INSTABILITY OF A HOLLOW JET ENDOWED WITH SURFACE TENSION
}

\author{
Ahmed E. Radwan* and Nasreldein A. Ahmed ${ }^{\star *}$
}

\begin{abstract}
The higher orders instability of a gas cylinder ambient with an incompressible inviscid liquid endowed with surface tension is analyzed. The perturbation equations up to third order are derived and solved. The surface displacements, the velocity potentials and the dispersion relations are derived for each order of axisymmetric perturbation. It is found that, up to third order, a transition from instability to stability states occurs when the perturbed wavelength equals the circumference of the gas cylinder. The stability discussions for the present model have been done and for the nonhollow jet as well. The hollow jet instability is much larger than that of the nonhollow model. It is found that the maximum temporal amplification prevailing in the hollow jet is much higher than that of the full fluid jet. These results are consistent with some data of the experimental work of Kendall (1986) phys. Fluids 29, 2086, in the first order perturbation.
\end{abstract}

\section{KEY WORDS}

Nonlinear stability, Surface tension, Hollow jet

\footnotetext{
*Professor, Department of Mathematics, Faculty of Science, Ain-Shams University, Cairo, Egypt.

${ }^{* *}$ Engineer, Department of Basic Science, Benha Higher Institute of Technology, Benha, Egypt.
} 


\section{INTRODUCTION}

In the last decades some studies have examined the instability of a full jet surrounded by a vacuum (see Plateau (1973), Rayleigh (1945), Lamb (1945), Drazin \& Reid (1980), Chandrasekhar (1981) and Radwan (1995)). Kendall (1986) performed an experimental study of the instability of annular jets in application to astrophysics. Radwan has discussed the capillary instability of a ((1999), (2001) and (2005)) hollow jet under different forces. In separate studies Chandrasekhar (1981) determined the dispersion of non-viscous, non-streaming hollow cylinder for small axisymmetric perturbations and deduced that the unstable waves frequency $\omega$ grow in according with

$$
\omega^{2}=\frac{T}{\rho a^{3}} \frac{x K_{1}(x)}{K_{o}(x)}\left(x^{2}-1\right) .
$$

Here $\rho$ and $T$ are the liquid mass density and surface tension coefficient, $x \quad(=k a$, where $k$ is the ordinary wavenumber) is the non-dimension longitudinal wavenumber, $a$ is the gas jet radius, $K_{1}(x)$ and $K_{o}(x)$ are the modified Bessel functions of second kind of the first and zero order, respectively.

The present work investigates the higher orders instability of a hollow jet (a gas cylindrical jet embedded in a liquid) due to the interaction of the capillary and inertia forces where the liquid inertia is greater than that of the gas.

One has to mention here that the phenomenon of a hollow jet may occur in nature when a gas escaping from below a liquid layer. For instances in the crust of the earth when a gas escapes from below an oil layer or in the sea during geological drillings or when the air is pumped into a fluid layer.

\section{MATHEMATICAL FORMULATION}

Consider cylindrical interface of radius $a$, immersed in an inviscid and incompressible liquid extending radially to infinity. A cylindrical polar coordinates $(r, \theta, z)$ with z-axis coinciding with the axis of the cylinder will be utilized. The curvature pressure due to surface tension cf. Radwan (1989), $P_{s}$, at the gasliquid interface $S$ is

$P_{s}=-T\left(\nabla \bullet \underline{n}_{s}\right)$,

where $\underline{n}_{s}(=\nabla F /|\nabla F|)$ is a unit outward vector pointing from gas to liquid region and $F(r, \theta, z ; t)=0$ describe the surface of liquid element at time $t$. The distribution of the equilibrium $P_{o}$, is given by

$$
P_{o}=P_{g}-\frac{T}{a}
$$


The second term in the right side of (2) represents the contribution due to surface tension while $P_{g}$ is a gas constant pressure: the hollow cylinder has to be filled by a gas of constant pressure to insure the equilibrium state. Clearly $P_{g}$ must be greater than $T / a$ otherwise the configuration will collapse towards a hollow cylinder of smaller radius to reach an equilibrium state.

We next consider perturbation of the equilibrium state using a linearized theory to that one in description of the break-up of the hollow cylinder. Since the hollow cylinder can be unstable for certain wavelengths for the rotationally symmetric modes, so non-axisymmetric modes will not be considered. All physical variables are normalized with respect to the characteristic length, $a$ (the radius of the undisturbed column) and the characteristic time $\left(T / \rho a^{3}\right)^{-(1 / 2)}$. At the initial state, we impose at the gas-liquid surface: rotationally sinusoidal perturbation of longitudinal wavenumber $k$ and amplitude $\eta_{o}$ where $\eta_{o}<<1$. Next we assumed that the column response only to the action of the surface tension, all other effects are assumed to be negligible. We also assume that the flow of the fluid is remaining irrotational and axisymmetric as in the initial state, since the irrotational motion will persist, see Drazin \& Reid (1981). So that the motion can be described by the velocity potential $\phi(r, z ; t)$ which satisfies Laplace's equation

$\nabla^{2} \phi=0$,

at the free surface

$r=1+\eta(z, t)$.

Here $\eta$ is the non-dimension surface wave amplitude as related to the undisturbed state. At $t=0$, we impose

$\eta(z, 0)=\eta_{o} \cos (k z)+\left(1-\frac{1}{2} \eta_{o}^{2}\right)-1$,

and

$\frac{\partial \eta(z, 0)}{\partial t}=0$

Equation (3) is subjected to boundary conditions

$\left[\frac{\partial}{\partial t}+(\nabla \phi \bullet \nabla)\right] \eta=\frac{\partial \phi}{\partial r}$,

and

$\frac{\partial \phi}{\partial t}+\frac{1}{2}\left(\left(\frac{\partial \phi}{\partial r}\right)^{2}+\left(\frac{\partial \phi}{\partial z}\right)^{2}\right)$ 


$$
=(1+\eta)^{-1}\left[1+\left(\frac{\partial \eta}{\partial z}\right)^{2}\right]^{-\frac{1}{2}}-\frac{\partial^{2} \eta}{\partial z^{2}}\left[1+\left(\frac{\partial \eta}{\partial z}\right)^{2}\right]^{-\frac{3}{2}}
$$

at the free surface (4). The velocity potential $\phi$ and the surface amplitude $\eta$ are then expanded as a perturbation series in terms of $\eta_{o}(<<1)$ :

$$
\begin{aligned}
& \phi(r, z ; t)=\sum_{n=1} \eta_{o}^{n} \phi_{n}(r, z ; t), \\
& \eta(z ; t)=\sum_{n=1} \eta_{o}^{n} \eta_{n}(z ; t) .
\end{aligned}
$$

By the use of the expansions (9) and (10) for (3) and (5)--(8) then expanding by utilizing Taylor series around the undisturbed interface surface $(r=1)$ and equating the terms of equal powers $\eta_{o}$, one obtains a sequence of sets of equations for $\phi_{n}$ and $\eta_{n}$.

\section{First order}

$\nabla^{2} \phi_{1}=0$,

$\phi_{1, r}-\eta_{1, t}=0, \quad$ at $r=1$,

$\phi_{1, t}+\eta_{1}+\eta_{1, z z}=0, \quad$ at $r=1$,

$\eta_{1}(z, 0)=\cos k z$,

$\eta_{1, t}(z, 0)=0$

Second order

$\nabla^{2} \phi_{2}=0$

$\phi_{2, r}-\eta_{2, t}=\eta_{1, z} \phi_{1, z}-\eta_{1} \phi_{1, r r}$,

at $r=1$,

$\phi_{2, t}=-\frac{1}{2}\left(\phi_{1, r}^{2}+\phi_{1, z}^{2}\right)+\eta_{1}^{2}-\eta_{2}-\eta_{2, z z}+\eta_{1} \phi_{1, t r}, \quad$ at $r=1$,

$\eta_{2}(z, 0)=-\frac{1}{4}$

$\eta_{2, t}(z, 0)=0$,

Third order

$\nabla^{2} \phi_{3}=0$

$\phi_{3, r}=-\eta_{3, t}+\eta_{2} \phi_{1, r r}+\eta_{1} \phi_{2, r r}+\frac{1}{2} \eta_{1}^{2} \phi_{1, r r r}-\eta_{2, z} \phi_{1, z}-\eta_{1, z}\left(\eta_{1} \phi_{1, r z}+\phi_{2, z}\right), \quad$ at $r=1$.

$\phi_{3, t}=-\eta_{3, z z}+\frac{3}{2} \eta_{1, z}^{2} \eta_{1, z z}+2 \eta_{1} \eta_{2}-\eta_{1}^{3}+\eta_{2} \phi_{1, t r}+\frac{1}{2} \eta_{1}^{2} \phi_{1, t r r}$

$$
+\eta_{1} \phi_{2, t r}+\eta_{1} \phi_{1, r} \phi_{1, r r}+\phi_{1, r} \phi_{2, r}+\eta_{1} \phi_{1, z} \phi_{1, z r}+\phi_{1, z} \phi_{2, z}, \quad \text { at } r=1 \text {. }
$$

$\eta_{3}(z, 0)=0$,

$\eta_{3, t}(z, 0)=0$, 
The problem is solved in each order, starting with the lowest one. The system of the first perturbation equations (11)--(15) is solved and are consistent with the classical results of Chandrasekhar (1981) and Radwan (1989):

$\eta_{1}=\cosh \left(\omega_{1} t\right) \cos (k z)$

$\phi_{1}=\omega_{1} \frac{K_{o}\left(k_{r}\right)}{k K_{1}(k)} \sinh \left(\omega_{1} t\right) \cos (k z)$

where

$\omega_{1}^{2}=\frac{k\left(1-k^{2}\right)}{K_{a}}, K_{a}=K_{o}(k) / K_{1}(k)$.

The solution of the second order perturbation eouations is solved on utilizing the system of equation (16)--(20). Assuming that the second order perturbation of the dimensionless surface wave amplitude $\eta_{2}(z ; t)$ is of the form

$\eta_{2}(z ; t)=B_{22}(t) \cos (2 k z)+D_{2}(t)$.

Here $B_{22}(t)$ is an arbitrary function of time to be determined, while $D_{2}(t)$ is (required to insure a conservation of mass at $t>0$ such that $\eta$ satisfies (19)) given by

$D_{2}(t)=-\frac{1}{8}\left[1+\cosh 2 \omega_{1} t\right]$

Using the $z$-dependence for the Laplacian equation (16) then, apart from the singular solution, $\phi_{2}$ is determined

$\phi_{2}=A_{2}(t) K_{o}(2 k r) \cos 2 k z+F_{2}(t)$,

where $A_{2}(t)$ and $F_{2}(t)$ are some functions of time. By using equations (26)--(30) for equation (17) and equating the coefficients of $\cos 2 k z$ and those which are independent of $z$; we find that $A_{2}(t)$ is given by

$$
A_{2}(t)=\frac{1}{2 k K_{1}(2 k)}\left[\dot{B}_{22}(t)+P_{22} \sinh 2 \omega_{1} t\right]
$$

where dot over the variables indicates the differentiation with respect to the argument and $P_{22}$ is defined by

$$
P_{22}=\omega_{1}\left(1-2 k K_{a}\right) / 4
$$

By substitution of equations (26)--(32) into equation (18) and identify the coefficients of the trigonometric functions which occurred there, yields 


$$
F_{2}=B_{2} t+C_{2} \sinh 2 \omega_{1} t
$$

such that

$$
\begin{aligned}
& B_{2}=-\frac{1}{8}\left[3+\omega_{1}^{2}\left(1-K_{a}^{2}\right)\right], \\
& C_{2}=-\frac{1}{16 \omega_{1}}\left[3+\omega_{1}^{2}\left(3+K_{a}^{2}\right)\right],
\end{aligned}
$$

Moreover, the identification of the coefficients which are independent of $z$ in the expression resulting from the substitution of (26)--(32) into (18) degenerates to

$$
\begin{aligned}
& B_{22}=a_{22} \cosh \left(\omega_{2} t\right)+b_{22} \cosh \left(2 \omega_{1} t\right)+c_{22}, \\
& b_{22}=\frac{1}{4\left(\omega_{2}^{2}-4 \omega_{1}^{2}\right)}\left[2 \omega_{1}^{2}\left(1-2 k K_{a}\right)+\frac{k\left(2+\omega_{1}^{2}\left(3-K_{a}^{2}\right)\right)}{K_{b}},\right. \\
& c_{22}=\frac{k}{4 \omega_{2}^{2} K_{b}}\left[2+\omega_{1}^{2}\left(1+K_{b}^{2}\right)\right],
\end{aligned}
$$

and

$$
\omega_{2}^{2}=\frac{2 k\left(1-k^{2}\right)}{K_{b}}=\omega_{1}^{2}(2 k),
$$

where $K_{b}$ is defined as

$$
K_{b}=K_{o}(2 k) / K_{1}(2 k) .
$$

By substituting from equations (36), (29) and (28) into equation (19), the coefficient $a_{22}$ is given as

$$
a_{22}=-\left(b_{22}+c_{22}\right)
$$

Following the same analysis as that of second order; the solution corresponding to the third order perturbation can be obtained by the use of the first and second orders and the system of equations (21)...(25). In view of (28), the third order amplitude $\eta_{3}(z, t)$ can be assumed of the form

$\eta_{3}(z, t)=B_{33}(t) \cos (3 k z)+D_{3}(t)$ 
where $D_{3}(t)=0$ in order to satisfy the conservation of mass and that (42) satisfies (24). Using the z-dependence (see (42)), solving (21) and applying the kinematics boundary condition (22) (under the present circumstances) we see that $\phi_{3}(r, z, t)$ must be

$$
\phi_{3}=P_{31}(t) \frac{K_{o}(k r)}{k K_{1}(k)} \cos k z+\left[P_{33}(t)+B_{33}(t)\right] \frac{K_{o}(3 k r)}{3 k K_{1}(3 k)} \cos 3 k z
$$

which is a finite solution as $r$ tends to infinity.

The use of the continuity condition (23) of the normal component of the total stress tensor across the boundary surface at the equilibrium position $(r=1)$ gives

$$
\begin{aligned}
B_{33}(t)= & b_{330} \cosh \left(\omega_{3} t\right)+b_{331} \cosh \left[\left(\omega_{2}+\omega_{1}\right) t\right]+b_{332} \cosh \left[\left(\omega_{2}-\omega_{1}\right) t\right]+ \\
& +b_{333} \cosh 3 \omega_{1} t+b_{334} \cosh \left(\omega_{1} t\right)
\end{aligned}
$$

and yields

$\omega_{3}^{2}=\frac{3 k\left(1-9 k^{2}\right)}{K_{c}}=\omega_{1}^{2}(3 k)$,

where

$$
K_{c}=K_{o}(3 k) / K_{1}(3 k)
$$

and where $b_{33 i}(i=0,1, \ldots ., 4) ; P_{31}$ and $P_{33}$ are given in the appendix.

Clearly from the dispersion relations (45) and (39) for the third and second order perturbations, the cut-off wavenumber remains unity as is found as in the linearized theory of Chandrasekhar (1981).

\section{DISCUSSIONS AND CONCLUSION}

Here we have obtained the capillary instability criteria of a gas jet (of negligible inertia) immersed in a liquid for the orders third, second and first. These criteria are found to be the same in all orders of perturbations. We mean that the cut-off wavenumber i. e. the point at which a transition from oscillation to instability states occurred, is the same (i. e. $k=1$ ) in the linear and nonlinear perturbations. This occurs as the perturbed wavelength is equal to the circumference of the gas cylinder. This behavior is also the same even in the case of full liquid jet ambient with a gas of totally negligible influence. Also we found here the gas constant pressure of the gas cylinder has no direct influence on the stability of the hollow jet but just to cope with the negative pressure due to the curvature of the gas-liquid interface in the unperturbed state to maintain and keep the existence of the model. 
The analytical and numerical analyses of the stability criteria have been shown that the stability domain is $0<k<1$ and that of stability is being $1 \leq k<\infty$ where $k=1$ corresponds to $(\omega=0)$ the marginal stability. The maximum mode of instability is found to be 0.8201 (at $k=0.484$ ) which is in fact too much larger than that of the full liquid jet in vacuum that found to be 0.3433 (at $k=0.697$ ) (see Chandrasekhar's figure $3, p$ 541. It is found that the hollow cylinder is also much more stable than the nonhollow jet not only in the axisymmetric domain $1<k<\infty$ but also even in the nonaxisymmetric domain $0<k<\infty$ see Radwan (1989) for linear analysis.

Using the properties and behaviour of the Bessel functions (cf. Abramowitz and Stegun (1970)) as $k$ is too much less than unity i. e. for very long wavelengths, we get

$\omega^{2}=\left(\ln \frac{\not k}{2}\right)^{-1}+\ldots .$.

where $(\ln \gamma)$ is the Eulerian constant. Moreover, as $k \rightarrow 0$, we get

$\omega^{2}=\frac{-1}{k^{2} \ln k}+\ldots$

We deduce that the temporal amplification tends to zero with vertical tangent as $k \rightarrow 0$, in contrast for the full liquid jet in vacuum the temporal amplification approaches zero linearly as $k \rightarrow 0$.

In general case for any value of $k$, the temporal amplification pervaded in the hollow case is much higher than that pervading in the nonhollow liquid jet, see Kendall (1986) (p. 2086). 
APPENDIX

The coefficients $b_{33 i}(i=0,1,2, \ldots, 4) ; b_{31}$ and $b_{33}$ which are carried in equations (44) and (43) are given explicitly as following.

First $b_{33 i}(i=0,1,2, \ldots, 4)$ are

$$
\begin{aligned}
& b_{330}=-\sum_{i=1}^{4} b_{33 i}, \\
& b_{331}=\frac{1}{\left(S_{1}^{2}-\omega_{3}^{2}\right) K_{c}}\left[3 k q_{331}-S_{1} P_{331} K_{c}\right], \\
& b_{332}=\frac{1}{\left(S_{2}^{2}-\omega_{3}^{2}\right) K_{c}}\left[3 k q_{332}-S_{2} P_{332} K_{c}\right], \\
& b_{333}=\frac{1}{\left(9 \omega_{1}^{2}-\omega_{3}^{2}\right) K_{c}}\left[3 k q_{333}-3 \omega_{1} P_{333} K_{c}\right], \\
& b_{334}=\frac{1}{\left(\omega_{1}^{2}-\omega_{3}^{2}\right) K_{c}}\left[3 k q_{334}-\omega_{1} P_{334} K_{c}\right],
\end{aligned}
$$

where $b_{33 i}(i=0,1,2, \ldots, 4)$ will be written down later on while $S_{1}, S_{2}$ and $b_{33 i}$ $(i=0,1,2, \ldots ., 4)$ are defined as

$$
S_{1}=\omega_{2}+\omega_{1}, S_{2}=\omega_{2}-\omega_{1},
$$

and

$$
\begin{aligned}
q_{331}= & -\frac{a_{22}}{4}\left[2+S_{1}^{2}-\omega_{1} \omega_{2}\left(1+K_{a} K_{b}\right)\right], \\
q_{332}= & -\frac{a_{22}}{4}\left[2+S_{2}^{2}+\omega_{1} \omega_{2}\left(1+K_{a} K_{b}\right)\right], \\
q_{333}= & \frac{1}{32}\left[2-3 k^{4}+\omega_{1}^{2}\left(3-k K_{a}\right)-8 b_{22}\left(2+\omega_{1}^{2}\right)+\right. \\
& \left.+8 \omega_{1}^{2}\left(2 b_{22} \omega_{1}+P_{22}\right)\left(K_{a} K_{b}-3\right)\right], \\
q_{334}= & \frac{1}{32}\left[6+9 k^{4}+\omega_{1}^{2}\left(1-3 k K_{a}\right)-8 \omega_{1}\left(2 b_{22} \omega_{1}+P_{22}\right)\left(1+K_{a} K_{b}\right)-\right. \\
& \left.-8\left(2+\omega_{1}^{2}\right)\left(b_{22}+c_{22}\right)\right],
\end{aligned}
$$


The coefficients $P_{31}(t)$ and $P_{33}(t)$ are given by

$P_{31}=P_{311} \sinh \left(S_{1} t\right)+P_{312} \sinh \left(S_{2} t\right)+P_{313} \sinh \left(3 \omega_{1} t\right)$,

$P_{33}=P_{331} \sinh \left(S_{1} t\right)+P_{332} \sinh \left(S_{2} t\right)+P_{333} \sinh \left(3 \omega_{1} t\right)+$

$+P_{334} \sinh \left(\omega_{1} t\right)$,

where the coefficients $b_{31 i}$ and $b_{33 i}$ (with $i=1,2,3,4$.) are defined by

$$
\begin{aligned}
P_{311}= & \frac{a_{22}}{4}\left[S_{1}+k\left(\omega_{1} K_{a}-\omega_{2} K_{b}\right)\right], \\
P_{312}= & \frac{a_{22}}{4}\left[S_{2}+k\left(\omega_{2} K_{b}-\omega_{1} K_{a}\right)\right], \\
P_{313}= & \frac{1}{32}\left[8 \omega_{1} b_{22}\left(k K_{a}-2 k K_{b}+3\right)+5 \omega_{1} k K_{a}+8 P_{22}\left(1-k K_{b}\right)-\omega_{1}\left(8+k^{2}\right)\right], \\
P_{314}= & \frac{\omega_{1}}{32}\left[5 k K_{b}-k^{2}+8\left[-1+\left(1-k K_{b}\right)\left(2 b_{22}+P_{22} / \omega_{1}\right)-\right.\right. \\
& \left.\left.-\left(1+k K_{a}\right)\left(b_{22}-2 c_{22}\right)\right]\right],
\end{aligned}
$$

and

$$
\begin{aligned}
P_{331}= & \frac{a_{22}}{4}\left[S_{1}-3 k\left(\omega_{1} K_{a}+\omega_{2} K_{b}\right)\right], \\
P_{332}= & \frac{a_{22}}{4}\left[S_{2}+3 k\left(\omega_{1} K_{a}-\omega_{2} K_{b}\right)\right], \\
P_{333}= & \frac{\omega_{1}}{32}\left[-2-3 k^{2}+k K_{a}+8\left(1-3 k K_{b}\right)\left(2 b_{22}+P_{22} / \omega_{1}\right)+\right. \\
& \left.+8 b_{22}\left(1-3 k K_{a}\right)\right], \\
& \\
P_{334}= & P_{333}-\frac{\omega_{1}}{2}\left(b_{22}-c_{22}\right)\left(1-3 k K_{a}\right) .
\end{aligned}
$$




\section{REFERENCES}

[1] Abramowitz, M. and Stegun, I., Handbook of Mathematical Functions, Dover Publ., New York, USA, (1970).

[2] Chandrasekhar, S., Hydrodynamic and Hydromagnetic Stability, Dover Publ., New York, USA, (1981).

[3] Chen, F., Tsaur, J., Durst, F. and Das, S., "on the Axisymmetry of Annular Jet Instabilities" J. Fluid Mech., Vol. 448, PP 355, (2003)

[4] Drazin, P. and Reid,W., Hydrodynamic Stability, Cambridge Univ. Press, London, (1985)

[5] Kendall, J., "Experiments on Annular Liquid Jet Instability and on the Formation of Liquid Shells" Phys. Fluids, Vol. 29, PP 2086, (1986).

[6] Lamb, H., Hydrodynamics, Dover Publ., New York, USA, p 473, (1945).

[7] Lee, C. and Wang, T., "the Theoretical Model for the Annular Jet Instability" J. of Phys. Fluids, Vol. A1, PP 967, (1989).

[8] Meyer, J. and Weihs, D., "Capillary Instability of an Annular Liquid Jet"J. of Fluid Mech., Vol.179, PP 531, (1987).

[9] Plateau, J., " Statique Experimentale et Theorique des Liquides Soumis aux Seules Forces Moleculaires" J. of Gauthier-Villars, Paris, France, Vols. 1 and 2, (1873).

[10] Radwan, A., "Instability of a Hollow Jet with Effects of Surface Tension and Fluid Inertia" J. of Phys. Soc. Japan, Vol. 58, PP 1225, (1989).

[11] Radwan, A., "Capillary Gravitodynamic Instability of a Two Fluids Interface" J. of Phys. Scripta., Vol. 51, PP 484, (1995).

[12] Radwan, A. and Gad, N., "Electrification Capillary stability of a Hollow Jet "J. of Arch. Mech., Vol. 51, PP 633, (1999).

[13] Radwan, A. and Sileem, S., "Hydromagnetic Instability Streaming HollowJet with Perturbed Interfaces" J. of Chaos, Solitons and Fractals, Vol.12, PP 1415, (2001).

[14] Radwan, A. and Alkaabi, A., "Hydromagnetic Capillary stability of Streaming Hollow Cylinder with Azimuthal Field Interface" J. of Mech. \& Mechanical Energy, Vol. 8, PP 163, (2005).

[15] Rayleigh, J., The Theory of Sound, Dover Publ., New York, USA, (1945). 